\title{
Network Compatibility Blocs as Basis of Partner's Selection
}

\author{
Salah Zouggar, Marc Zolghadri, Xin Zhang, and Philippe Girard \\ IMS - LAPS/GRAI, University of Bordeaux, UMR 5218CNRS \\ 351, Cours de la libération, 33405 Talence Cedex, France \\ \{Salah.Zouggar, Marc.Zolghadri, Xin. Zhang, \\ Philippe.Girard\} @ims-bordeaux.fr
}

\begin{abstract}
The aim of this paper is to contribute to partner's network design beyond the common partner's selection process usually made in the literature. The originality of our approach lies in compatibility concept that comes to consolidate the ordinary approach of partner's selection. We suggest the use of product architecture to extract its related network of partners that would be analyzed with paying attention not only to the efficiency of each required partner within the network, but also to its compatibility with other actors. The gBOMO (generalised Bill Of Materials and Operations) concept becomes significant tool that we intensively use in order to detect the imperatives of realization phase of manufactured product. We will develop exploratory ideas about the network compatibility blocs. These ideas allow a better understanding of partner's compatibility requirements within network.
\end{abstract}

Keywords: Network design, product architecture, partner selection.

\section{Introduction}

The partners' selection was extensively studied in the scientific literature since several decades see for instance [1]. However, still remains the need for those firms, called Focal Company (FC), which initiate a product development project to improve their understanding of partnership and the relevancy of their partner selection procedures.

The partner's selection process for any company requires much more attention and know how than what was required in the past [2]. Indeed, the complexity of products and their components is growing everyday. This induces companies to be careful about their partners [3]. Nevertheless, partner's selection is highly risky because of their weaknesses could be propagated to the whole network.

The architecture of the partners' network is not independent from the product that it aims to manufacture. Starting from this observation, we claim that simultaneous analysis of product architecture and network architecture is of upmost importance for the selection process of relevant partners. This could be done by considering partners potential exchanges and/or dependencies. 
The paper is organized as follows. In section 2 a brief state-of-the-art reviews some existing works in the field of product and network modularity. In section 3, we evoke the product architecture and explore its links with the associated partners' network architecture. Section 4 treats the partners' selection process. In section 5, network compatibility blocs approach is developed. Finally, some conclusions and perspectives end the paper.

\section{Related Works}

The architecture of any product can be more or less modular. In recent years, several author's publications see benefits in adopting modular product design [4], [5], [6]. In fact, in modular product architecture, components are interchangeable, autonomous, loosely coupled, and individually upgradeable thanks to standardized interfaces [7]. In this case, mapping between functions and components becomes easier. For a modular product, two major factors are: dependence of components and their interfaces. The dependence refers to the way that one component needs some inputs coming form another while the interface defines precisely how this exchange is performed. Obviously, exchanges between components could be and are, quite frequently bilateral.

Focusing on the network, the modularity is measured through proximity concept defined by [7] along the following dimensions: 1- geographic proximity that can be measured by physical distance; 2 - organizational proximity which deals with ownership, managerial control and interpersonal and inter-team interdependencies, 3- cultural proximity that captures commonality of language, business mores, ethical standards and laws, among other things, and finally, 4- electronic proximity that can be captured by e-mail, EDI, intranets, video conferencing, etc. These measures are of great indicators for network modularity. But, here authors consider hat they are not enough to characterise exactly the network. The complementarity and dependence of partners are considered to be of high importance.

\section{Network Architecture Extracted from Product Architecture}

To clearly define these concepts, we use the gBOMO, generalised Bill Of Materials and Operations, developed in [8], adapted from Jiao BOMO [9]. This representation gathers jointly technical data of BOO (Bill Of Operations) and BOM (Bill Of Materials) of a considered product. gBOMO looks for perceiving the connection between the focal company and a subset of its major partners (cf. Fig 1).

gBOMO allows representing the realization phase as workflows involving partners at various stages. This highlights the exchanges which should take place from one partner to another directly (if they should collaborate together) or indirectly (their collaboration is structured through the FC). 


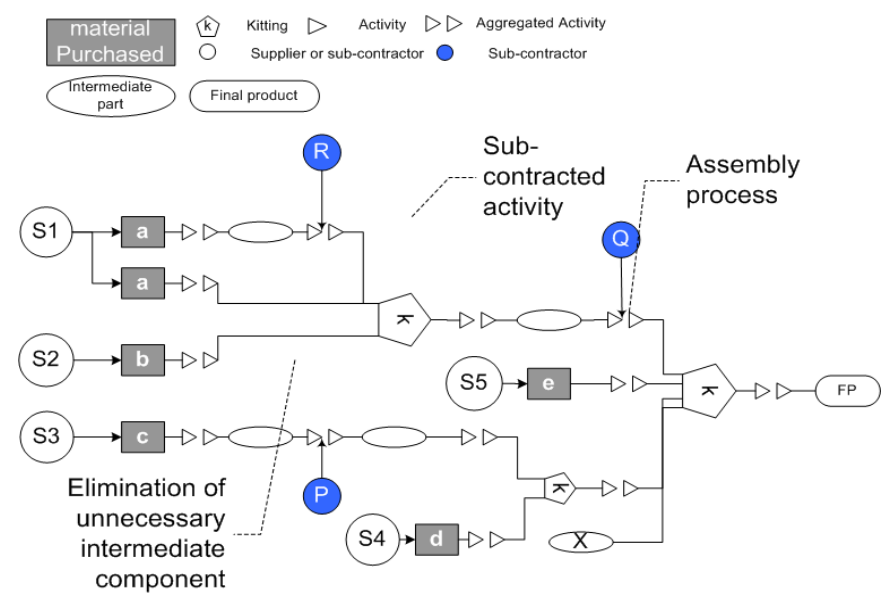

Fig. 1. gBOMO of product

\section{Partner's Selection in the Network}

Our analysis on partner's selection process is based on information got from gBOMO, we quote the number of partners, the adjacent partners and the kind of relationship (subcontractor, supplier, service providers....)

gBOMO allows a visual understanding of executive operations of manufacturing product during realization phase to perceive those partners that are directly involved in the same workflow. Therefore, hereafter we talk about dyadic relationships to start gradually our analysis on neighbor partners and extend the analysis to effectively and really linked partners in the network by highlighting hidden links.

\subsection{Dyadic Relationship}

Each couple of adjacent partners detected within the gBOMO might be dependent on their work contributions.

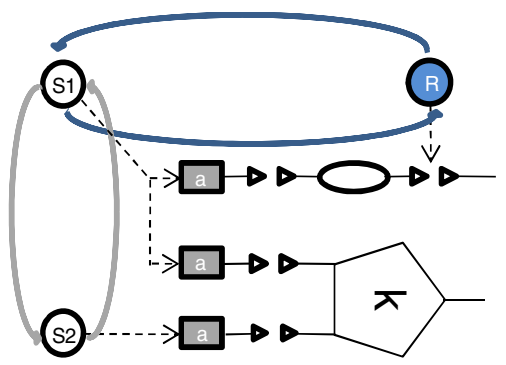

Fig. 2. Dyadic partners' relationship 
As shown in figure 2, we consider a couple of actors S1 and R. They operate in a sequential way with objective of adding value to the same workflow; the upstream or downstream position is relative to the workflow direction. Downstream Partner $\mathrm{R}$ has expectations and requirements on the work provided by upstream partner S1. As the upstream partner $\mathrm{S} 1$ is expected to add value to the workflow, its contribution can be evaluated according to his ability to satisfy the requirements of R. Ideally, these requirements and expectations must be fulfilled by contributions of upstream partner S1 at $100 \%$. If we consider the couple S1 and S2, they operate in parallel way, there is no direct dependency of their works, but the junction point $\mathrm{K}$ gathers their flows and generate a relative dependency between $\mathrm{S} 1$ and $\mathrm{S} 2$.

In the following points, we will focus on existing exchanges between partners during the realization phase, and suggest improvements to the selection process.

\subsection{Partner's Effectiveness}

Different criteria and indicators for partner's evaluation have been suggested throughout the literature. According to mostly known library of indicators of Huang and Keskar in [1], we achieve to distinguish two evaluation dimensions of partners:

1. Product-related effectiveness: that we define as the technical capability of a partner to design or realize given product parts.

2. Process-related effectiveness: it reflects the organizational capability of the partner and its ability to perform activities following the network dynamics.
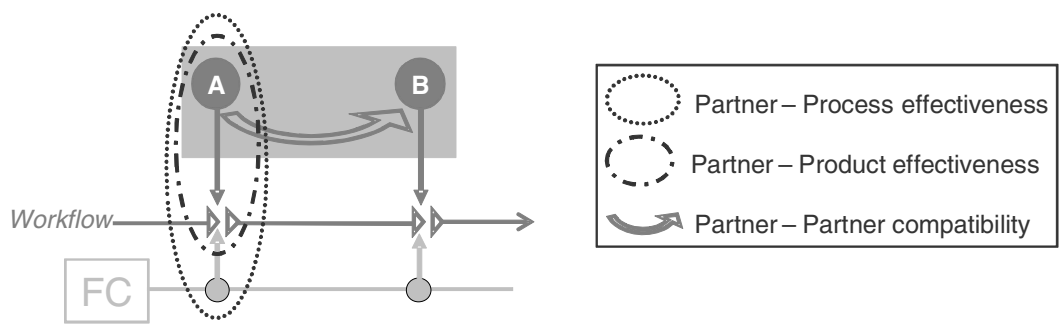

Fig. 3. Framework of compatibility analysis

The distinction made between the two suggested dimensions is that the partner process-related effectiveness underlines mainly the processing abilities of partners while the product-related effectiveness focuses on the technical aspects of the partner's abilities. In [10], we argue that overall performance of the partners' network is not only linked to product-related effectiveness and process-related effectiveness but it depends also closely to partner-partner compatibility. (see Figure. 3)

The compatibility is defined as an indicator that qualifies the collaboration efficiency between two actors and directly reliant on the nature of interactions and exchanges between partners.

The objective is the enhancement of compatibility of each couple of linked partners in the network. Once the technical and technological aspects of the product are guaranteed and the networking dynamics are ensured, it seems obvious that higher is the 
compatibility better could be the added value to the workflow and as a consequence better would be the performance recorded by the network.

Hereafter a categorization of collaborative situations corresponding to the effective networking is proposed.

\subsection{Categorization of Collaborative Situations}

Let us take two partners A and B belonging to the same workflow. Their contributions are considered as an added value expected by the focal company. To classify their coupled contributions, we suggest considering two dimensions. In one hand, partners' effectiveness (product and process related) and on the other hand their compatibility (exchanges and interactions quality). Potential collaboration situations are classified in Fig. 4.

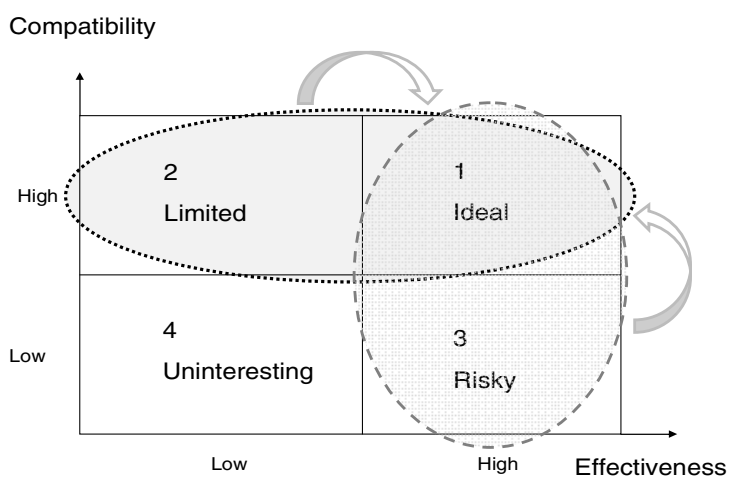

Fig. 4. Categorization of collaborative situations

Four collaboration categories maybe highlighted. They are ranked from the "ideal" collaboration situation (1) to the worst one (4):

1. Ideal. The category (1) corresponds to the ideal case, because the concerned partners have the best expected effectiveness and their compatibility is high. This situation has necessary but not sufficient conditions for optimal performance.

2. Limited. In category (2), partners have a low-level effectiveness but their compatibility is high. The use of this collaboration situation is limited and might be suitable only for non critical tasks.

3. Risky. In category (3), high-level effectiveness of partners is ensured but their ability to work together is not satisfying. This case is called risky because the highlevel of technicality could not guarantee by its own the success of the project.

4. Uninteresting. In category (4), partners have limited effectiveness and they are not efficient in working together. This case is uninteresting because dysfunctions could appear in both product and partners interactions.

As the expected objective of each company is to achieve the ideal case, the adaptation of categories (2) and (3) allows achieving that. 
Category (2) requires the improvement of the partners' effectiveness, this represents product and process oriented improvements which are often expensive to acquire, long to understand and difficult to implement.

For the third category (3), the effectiveness is proven; the only improvement to make is to enhance the compatibility which can be achieved, either by a better adaptation of the partners to the needs of each other, or by looking for new partners with expected effectiveness and a better compatibility.

In order to exploit these improvements based on compatibility, partners selection process has to be adapted and include a third dimension treating of partners links. These links are not always obvious and some of them could be hidden. Hereafter we focus on partners' dependencies to indentify the real links between all the partners of the network.

\section{Network Compatibility Blocs}

The study of the relationship between two adjacent partners as it was done until now seems obvious. In this section, the idea is to investigate relationships beyond not directly linked partners.

In [11] a dependency modeling approach has been proposed, the studied case concerns product made up five modules (a,b,c,d,e) and five partners within gBOMO (S1, S2, S3, S4, S5). This approach leads to linkage situations categorization by comparing product components linkage matrix and suppliers' strengths dependency matrix, (table 1).

Table 1. Linkage situations
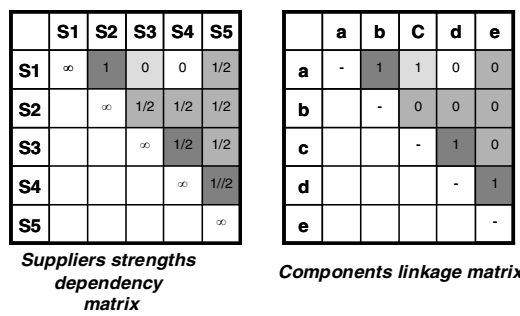

Components linkage matrix

\begin{tabular}{|l|l|l|}
\hline $\begin{array}{l}\text { Links types } \\
\text { Category }\end{array}$ & Supplier links & Components links \\
\hline Category 1 & Don't exist & Don't exist \\
\hline Category 2 & Don't exist & Exist \\
\hline Category 3 & Exist & Don't exist \\
\hline Category 4 & Exist & Exist \\
\hline
\end{tabular}

Based on the linkage situations, the first remark that we can make is the rising of two main situations: linked partners (directly or not) and not linked partners. According to this observation and the previous collaboration categorization of section 4.3 , we can deduce that categories $3 \& 4$ of partners linkage (linked partners) require considering the compatibility of corresponding partners while for categories $1 \& 2$, no compatibility is required among the unlinked partners.

\subsection{Blocs Extraction}

From supplier strength dependency matrix, we can identify for each partner the imperative compatible partners based on their link (table 2). We distinguish two kinds of links: the strong link (equals 1) and the weak one (less than 1). 
Table 2. Compatible partners' identification

\begin{tabular}{|c|c|c|c|c|c|c|c|c|}
\hline & s1 & S2 & S3 & s4 & S5 & Partner & Strong link & Weak link \\
\hline s1 & - & 1 & 0 & 0 & $1 / 2$ & S1 & S2 & S5 \\
\hline s2 & & - & $1 / 2$ & $1 / 2$ & $1 / 2$ & S2 & S1 & S3, S4, S5 \\
\hline s3 & & & 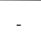 & $1 / 2$ & $1 / 2$ & S3 & & S2, S4, S5 \\
\hline S4 & & & & - & $1 / / 2$ & S4 & & S2, S3, S5 \\
\hline S5 & & & & & - & S5 & & $\mathrm{S} 1, \mathrm{~S} 2, \mathrm{~S} 3, \mathrm{~S} 4$ \\
\hline
\end{tabular}

Each group of partners where all the partners are linked constitutes a bloc (fig. 5). It is possible to identify subsets of partners, belonging to the network, which form network compatibility blocs because of their internal imperative synergy.
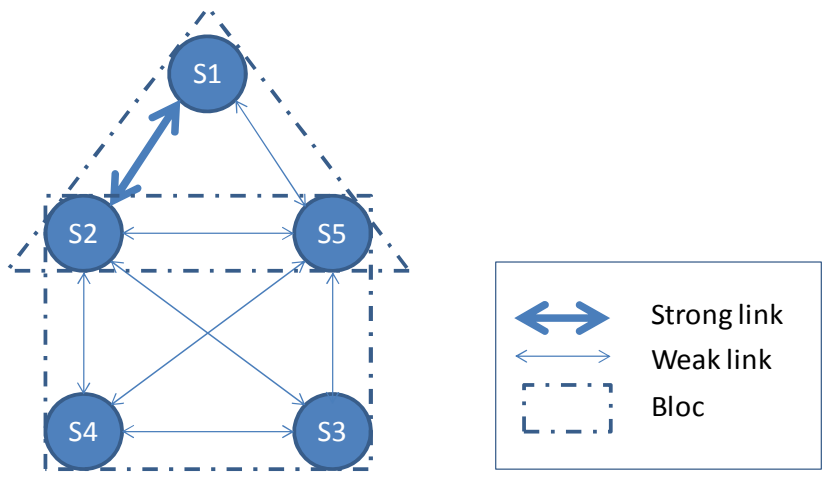

Fig. 5. Network compatibility blocs

Once all the blocs identified, partners' selection process can be improved by evaluating the compatibility in each bloc. This evaluation has to be done according to the kind of the links between partners and based on measures and criteria to be defined.

\section{Conclusion}

In a high versatile environment, the focal company needs more partnership flexibility to adapt its activities without creating costly dysfunctions. Thanks to suggested approach, for each product module and each new project, the FC would be able to select the suitable sub-contractors and suppliers, looking at the existing network. Modularity of product once correctly understood and coherently handled can brings significant benefits to the focal company, since it serves as support and basis to developing different groups of partners to carry out the most effective partnership and most flexible as possible.

Our analysis focused on the partners' compatibility improvements and partners' dependency links. As a result network compatibility blocs suggest new developments 
to adapt selection process and enlarge the scope to a third dimension of partners exchanges and relative dependencies that have to be considered specifically beyond the usual partners' selection process based on Product/Process related effectiveness.

Compatibility evaluation based on measures and criteria require a deep analysis of the linkage situations and necessitate further developments.

\section{References}

1. Huang, S.H., Keskar, H.: Comprehensive and configurable metrics for supplier selection. International Journal of Production Economics 105, 510-523 (2007)

2. Petersen, K.J., Handfield, R.B., Ragatz, G.L.: Supplier integration into new product development: coordinating product, process and supply chain design. Journal of Operations Management 23(3/4), 371-388 (2005)

3. Junkkari, J.: Higher product complexity and shorter development time continuous challenge to design and test environment. In: Design, Automation and Test in Europe Conference and Exhibition, pp. 2-3 (1999)

4. Gershenson, J.K., Prasad, G.J., Zhang, Y.: Product modularity: definitions and benefits. Journal of Engineering Design 14(3), 295-313 (2003)

5. Kusiak, A.: Integrated product and process design: a modularity perspective. Journal of Engineering Design 13(3), 223-231 (2002)

6. Ro, Y.K., Liker, J.K., Fixson, S.K.: Modularity as a strategy for supply chain coordination: the case of US auto. IEEE Transactions on Engineering Management 54(1), 172-189 (2007)

7. Fine, C.H.: Clockspeed: Winning Industry Control in the Age of Temporary Advantage. Perseus Books, Reading (1998)

8. Zolghadri, M., Baron, C., Girard, P.: Modelling mutual dependencies between products architecture and network of partners. International Journal of Product Development (2008) (to be printed)

9. Jiao, J., Tseng, M.M., Ma, Q., Zou, Y.: Generic Bill-of-Materials-and-Operations for high variety production management. CERA 4(8), 297-321 (2000)

10. Zouggar, S., Zolghadri, M., Girard, P.: Performance improvement in supply chains through better partners' selection. In: Proceedings of 13th IFAC Symposium on Information Control Problems in Manufacturing (2009)

11. Zouggar, S., Zolghadri, M., Girard, P.: Modelling product and partners network architectures to identify hidden dependencies. In: Proceedings of CIRP Design Conference (2009) 